\title{
Matrix Composition and Mechanics of Decellularized Lung Scaffolds
}

\author{
Thomas H. Petersen ${ }^{a, b}$ Elizabeth A. Calle ${ }^{b}$ Maegen B. Colehour ${ }^{b}$ \\ Laura E. Niklason ${ }^{\text {b, c }}$ \\ ${ }^{a}$ Department of Biomedical Engineering, Duke University, Durham, N.C., and Departments of \\ ${ }^{\mathrm{b}}$ Biomedical Engineering and ${ }^{\mathrm{C}}$ Anesthesiology, Yale University, New Haven, Conn., USA
}

\section{Key Words}

Decellularization $\cdot$ Tissue engineering $\cdot$ Matrix mechanics •

Collagen Elastin

\begin{abstract}
The utility of decellularized native tissues for tissue engineering has been widely demonstrated. Here, we examine the production of decellularized lung scaffolds from native rodent lung using two different techniques, principally defined by use of either the detergent 3-[(3-cholamidopropyl) dimethylammonio]-1-propanesulfonate (CHAPS) or sodium dodecyl sulfate (SDS). All viable cellular material is removed, including at least $99 \%$ of DNA. Histochemical staining and mechanical testing indicate that collagen and elastin are retained in the decellularized matrices with CHAPS-based decellularization, while SDS-based decellularization leads to loss of collagen and decline in mechanical strength. Quantitative assays confirm that most collagen is retained with CHAPS treatment but that about $80 \%$ of collagen is lost with SDS treatment. In contrast, for both detergent methods, at least $60 \%$ of elastin content is lost along with about $95 \%$ of native proteoglycan content. Mechanical testing of the decellularized scaffolds indicates that they are mechanically similar to native lung using CHAPS decellularization, including retained tensile strength and elastic behavior, demon-
\end{abstract}

strating the importance of collagen and elastin in lung mechanics. With SDS decellularization, the mechanical integrity of scaffolds is significantly diminished with some loss of elastic function as well. Finally, a simple theoretical model of peripheral lung matrix mechanics is consonant with our experimental findings. This work demonstrates the feasibility of producing a decellularized lung scaffold that can be used to study lung matrix biology and mechanics, independent of the effects of cellular components.

Copyright $\odot 2011$ S. Karger AG, Basel

\section{KARGER}

Fax +4161306 1234

E-Mail karger@karger.ch

www.karger.com
(C) 2011 S. Karger AG, Basel

1422-6405/12/1953-0222\$38.00/0

Accessible online at:

www.karger.com/cto
Dr. Laura E. Niklason

Departments of Biomedical Engineering and Anesthesia

Yale University, Room 301D Amistad Building

10 Amistad Street, New Haven, CT 06520 (USA)

Tel. +1 203737 1422, E-Mail laura.niklason@yale.edu 


\section{Introduction}

The principal determinants of the mechanical integrity of lung tissue are collagen and elastin fibers, although proteoglycans and cells can also contribute to tissue mechanics [Dunsmore and Rannels, 1996]. In this paper, we examine the contribution of these components to the mechanics of lung tissue by comparing native and decellularized lung tissues.

The decellularization of organs or tissues has been used to create material for tissue engineering applications, including heart valves [Bader et al., 1998], blood vessels [Dahl et al., 2003], and small-intestinal submucosa [Badylak et al., 1989]. The goal of the decellularization process is to remove cellular and nuclear material, while minimizing damage to the extracellular matrix (ECM). The development of a decellularized lung scaffold is valuable as a substrate for the growth of engineered lung tissue, as demonstrated in work from several laboratories [Cortiella et al., 2010; Ott et al., 2010; Petersen et al., 2010; Price et al., 2010]. In addition, a decellularized scaffold may be utilized to study the contributions of ECM components to the mechanical properties of lung tissue. Going further, purified lung ECM can be useful as a means to study substrate cues that impact pulmonary epithelial growth and differentiation.

There are many different types of decellularization protocols, each of which makes use of physical, chemical or enzymatic methods. Physical methods of decellularization, including freezing and vigorous agitation, could cause excessive concomitant destruction of the delicate lung ECM. Enzymatic methods alone, including DNase and other reagents, may not be cost-effective given the large volumes of liquid required for the treatment of an entire organ. Therefore, chemical treatments are utilized for most decellularization studies. The chemicals used in these studies were sodium chloride, sodium hydroxide, 3-[(3-cholamidopropyl)dimethylammonio]-1-propanesulfonate (CHAPS) or sodium dodecyl sulfate (SDS) and ethylenediaminetetraacetic acid (EDTA), which are all relatively low cost. A hypertonic sodium chloride solution can efficiently lyse cells via osmotic shock, although it does not assist in removing cellular components from the tissue [Gilbert et al., 2006]. CHAPS is a zwitterionic detergent, which allows efficient solubilization and thus removal of cellular material, particularly cellular and nuclear membranes [Dahl et al., 2003]. SDS is a highly ionic, amphipathic detergent that can disrupt cellular membranes and denature proteins [Gilbert et al., 2006]. EDTA is a chelating agent that binds key divalent ions (i.e. $\mathrm{Ca}^{2+}$ ) and aids in the disruption of cell attachment to the ECM via integrins [McFetridge et al., 2004]. In addition, the solution is of high alkalinity, which helps solubilize cytoplasmic cellular components as well as glycosaminoglycans (GAGs) which otherwise contaminate the matrix [Gilbert et al., 2006].

In this paper, we perform quantitative decellularization of rodent lungs using two different methods, and investigate the composition of decellularized lung tissues for collagen, elastin and proteoglycan content. We demonstrate that with CHAPS-based decellularization, collagen is retained, elastin is retained at about $40 \%$ of native levels, while GAGs are largely lost from the decellularized scaffolds. In contrast, with SDS-based decellularization, we find that collagen is retained at about $20 \%$ of native levels, elastin is retained at about $15 \%$ of native levels, while GAGs are also largely lost. Mathematical modeling studies helped to delineate the roles of collagen and elastin in the mechanics of the decellularized lung matrix. This work confirms that simple models for lung mechanics are fairly good at predicting lung ECM properties, and that lung matrices can provide a basis for future study of lung mechanics and lung tissue regeneration.

\section{Materials and Methods}

Organ Harvest and Decellularization

Lung tissue was obtained from young adult (3-month-old) male Fischer 344 rats. All animal experimental work was performed with approval from the Yale University Institutional Animal Care and Use Committee. Animals were euthanized with sodium pentobarbital and tissue was harvested. The lung tissue was decellularized for $4 \mathrm{~h}$ via vascular perfusion of $8 \mathrm{mM}$ CHAPS, $1 \mathrm{M} \mathrm{NaCl}, 25$ mM EDTA (prepared in $3 \mathrm{M} \mathrm{NaOH}, \mathrm{pH} 12$ ), and PBS, similar to a technique described previously [Petersen et al., 2010]. An alternate decellularization protocol was also evaluated, using $1 \mathrm{M} \mathrm{NaCl}, 25$ mM EDTA (prepared in $3 \mathrm{M} \mathrm{NaOH}$ ) and $1.8 \mathrm{~mm}$ SDS in PBS ('SDS decellularization'). Briefly, lungs were cannulated at the pulmonary artery trunk and perfused with decellularization fluids at $37^{\circ} \mathrm{C}$ through the vasculature with pressures kept below $30 \mathrm{~mm}$ $\mathrm{Hg}$. After decellularization, tissues were extensively rinsed via vascular perfusion with saline and analyzed as described below.

\section{Histological Analysis}

Tissues were formalin-fixed, paraffin-embedded and sectioned at $5 \mu \mathrm{m}$ thickness. Analysis was performed with standard hematoxylin and eosin staining, elastic van Gieson for elastin, and Alcian blue for proteoglycans, as well as staining for DNA using 4',6-diamidino-2-phenylindole (DAPI).

\section{Collagen Assay}

Collagen was quantified with a colorimetric assay to detect $\mathrm{OH}$-proline using a modification of Grant's [1964] method. Lung samples were lyophilized and weighed, then incubated in papain $(140 \mu \mathrm{g} / \mathrm{ml})$ at $60^{\circ} \mathrm{C}$ overnight (Sigma). Papain-digested samples 
Fig. 1. Diagram of model used to study tissue mechanics. A strip of lung tissue is modeled as $n$ elements in series. Each element is a spring (with spring constant $k$, modeling an elastin fiber) in parallel with a string (with length $l$, modeling a collagen fiber). A force $F$ is applied to the tissue, causing extension of the springs and elongation of the strings.

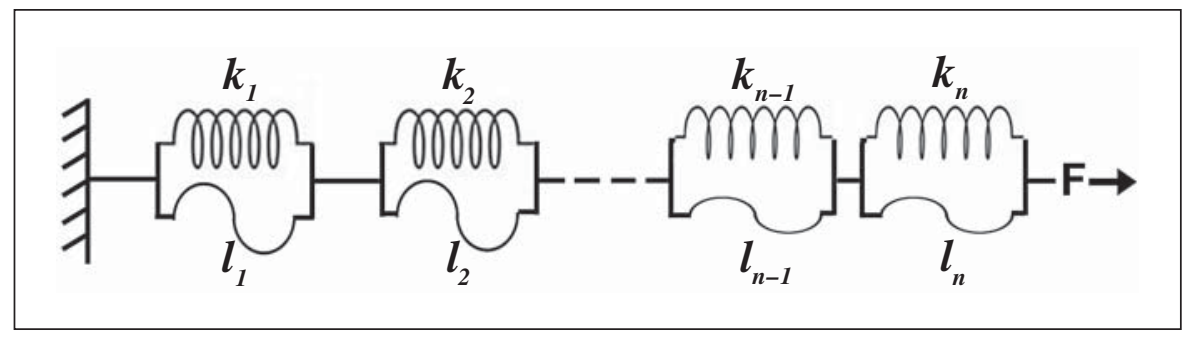

were incubated in $6 \mathrm{~N} \mathrm{HCl}$ at $115^{\circ} \mathrm{C}$ for $18 \mathrm{~h}$, neutralized, oxidized with chloramine-T and reacted with $p$-dimethylaminobenzaldehyde. Absorbance was measured at a wavelength of $550 \mathrm{~nm}$ and a $1: 10 \mathrm{w} / \mathrm{w}$ ratio of hydroxyproline to collagen was used to calculate the collagen content of the tissue. At least 4 samples were analyzed for native and decellularized samples.

\section{Elastin Assay}

Elastin was quantified using the Fastin Elastin assay kit (Biocolor, Belfast, N. Ireland). Lung samples were first lyophilized and weighed, and then the elastin was extracted following the method described in Foronjy et al. [2008]. Samples were incubated with $0.25 \mathrm{M}$ oxalic acid at $100^{\circ} \mathrm{C}$, then centrifuged at $10,000 \mathrm{~g}$ and the supernatant saved. The supernatant from 5 extractions was pooled, and the supernatant from the 6th extraction was also measured to ensure that no more elastin remained in the tissue. The oxalic acid was cleared using a 10,000 molecular weight cutoff filter (Millipore), then resuspended in $\mathrm{dH}_{2} \mathrm{O}$ and analyzed using the Fastin Elastin kit according to the manufacturer's instructions. At least 4 samples were analyzed for native and decellularized samples.

\section{Sulfated GAG Assay}

Sulfated GAGs were quantified using the Blyscan GAG assay kit (Biocolor). Papain-digested samples (prepared as described for the collagen assay, above) were assayed according to the manufacturer's instructions.

\section{DNA Assay}

DNA content of tissues was quantified using the Quant-iT PicoGreen dsDNA assay kit (Invitrogen, Eugene, Oreg., USA), following the manufacturer's instructions. Briefly, tissue samples were weighed and lyophilized, diluted in TE buffer and mixed with the Quant-iT PicoGreen reagent. Fluorescence was measured at $535 \mathrm{~nm}$ with excitation at $485 \mathrm{~nm}$, and DNA content was quantified using a standard curve.

\section{Mechanical Testing}

Native and decellularized lung samples were analyzed using an Instron 5848 equipped with a 10-newton load cell. Strips of tissue of known dimensions (typically 5-8 $\mathrm{mm}$ in length and 4-16 $\mathrm{mm}^{2}$ in cross-sectional area) were cut from peripheral areas of the lung, and were cyclically prestretched for 10 cycles to $20 \%$ strain to investigate elastic properties. Then, samples were stretched until failure to evaluate ultimate tensile strength (UTS). Using tissue dimensions, engineering stress and engineering strain were calculated from force and distance, where stress is the force divided by the initial cross-sectional area and strain is the distance divided by the initial length.

\section{Modeling}

We modeled the parenchymal pulmonary tissue using a simple linear model. The model is shown schematically in figure 1, where we model the system (a strip of lung tissue) as a linear series of elements, each of which is a spring in parallel with a string that has a defined maximum length (stop length). The string, which models collagen fibers, exerts no opposing force except when stretched to its stop length $l_{i}$, after which it cannot extend further. The spring models elastic fibers and behaves like a Hookean spring with constant $k_{i}$. This model is based on the description by Maksym and Bates [1997]. We apply this model to our system, and evaluate both the collagen and elastin contributions to the properties of decellularized scaffolds.

\section{Statistics}

Values are presented as means with standard error. Comparisons between groups were made using one-way analysis of variance with Bonferroni correction for multiple comparisons in GraphPad Prism.

\section{Results}

\section{Decellularization}

When rat lungs are subjected to decellularization as described, the decellularized scaffolds are completely devoid of any viable cells by standard histological techniques (fig. 2a, b). Despite loss of cellular material, there is maintenance of alveolar architecture at the light-microscopic level. Intact nuclei or cellular structures are not observed, although some elongated strands of DNA are occasionally noted with DAPI staining (fig. 2c, d). Quantitative DNA assay demonstrates the removal of at least 99\% of cellular DNA (table 1). Further analysis of CHAPSdecellularized lungs with transmission electron microscopy demonstrates that alveoli are intact with retained alveolar septa (fig. 2e, f), while microcomputed tomography and scanning electron microscopy have also demonstrated retained airway and vascular structure [Petersen et al., 2010]. Hence, the decellularized scaffolds are devoid of cellular material, yet maintain overall alveolar architecture and septal definition. 

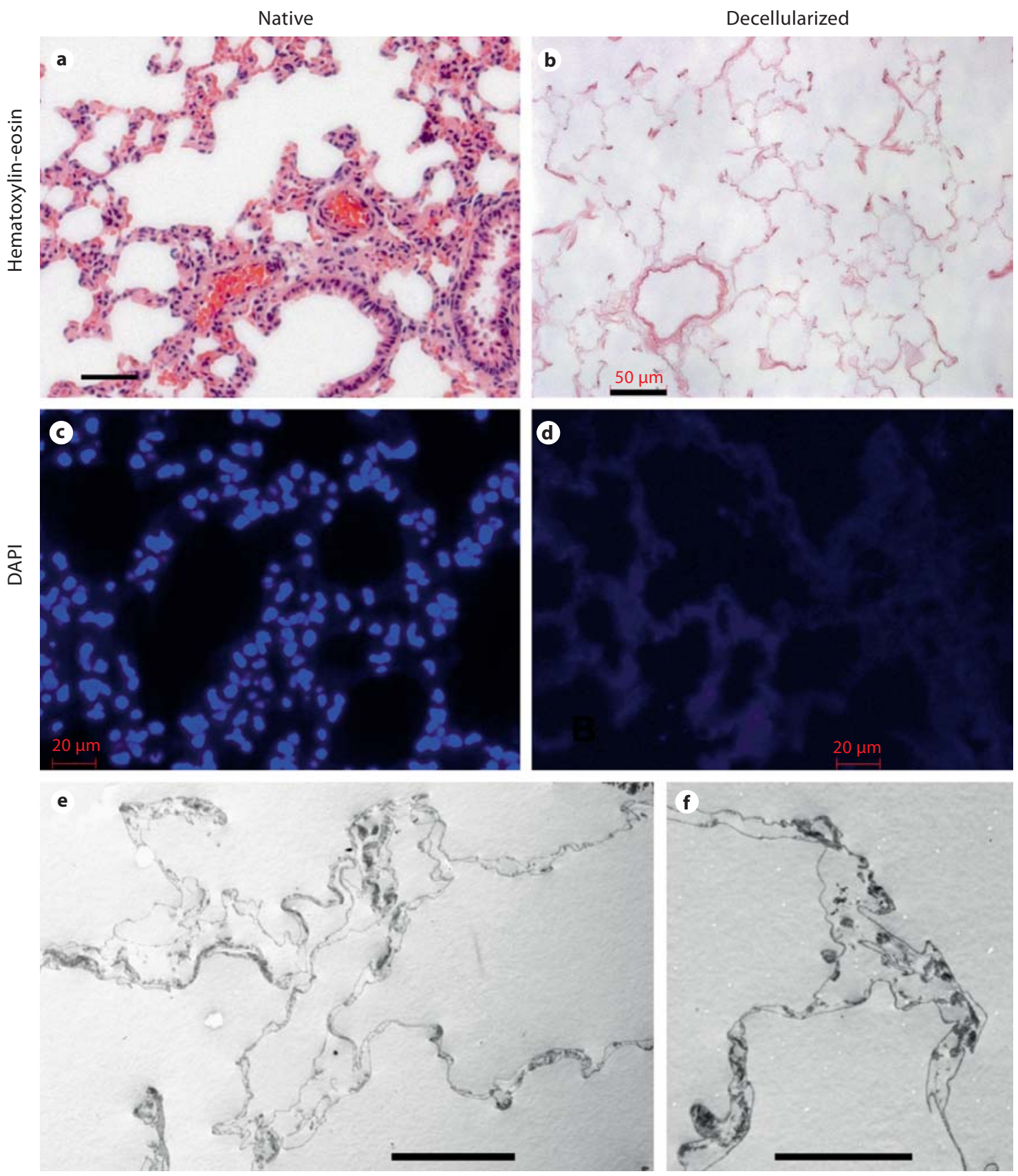

Fig. 2. Native and decellularized lung. a, b Hematoxylin and eosin histological stain of native (a) and decellularized (b) lung demonstrates loss of cellular and nuclear material after decellularization. Scale bars are $50 \mu \mathrm{m}$. c, d DAPI stain shows DNA in blue of native (c) and decellularized (d) lung and demonstrates loss of organized nuclear material in decellularized lung, although some faint DNA is distributed in the decellularized tissue. Scale bars are $20 \mu \mathrm{m}$. e-f Transmission electron microscopy of decellularized lung showing retained alveolar septa. Scale bars are $20 \mu \mathrm{m}$ in e and $10 \mu \mathrm{m}$ in $\mathbf{f}$. 
Table 1. Matrix characterization of acellular lung scaffolds

\begin{tabular}{lccc}
\hline & Native lung & Acellular lung & SDS-treated lung \\
\hline DNA, ng per lung & $2,370 \pm 100$ & $27.9 \pm 4.2^{*}$ & - \\
Collagen, $\mu$ g per lung & $3,480 \pm 103$ & $4,220 \pm 480$ & $787 \pm 102^{*}$ \\
Elastin, $\mu$ g per lung & $9,980 \pm 826$ & $3,760 \pm 291^{*}$ & $1,335 \pm 29.4^{*}$ \\
Sulfated GAGs, $\mu$ g per lung & $304 \pm 59$ & $19.1 \pm 3.37^{*}$ & $20.1 \pm 2.95^{*}$ \\
UTS, kPa & $19.8 \pm 2.4$ & $22.3 \pm 1.8$ & $7.48 \pm 1.5^{*}$ \\
\hline
\end{tabular}

Native and acellular lungs were characterized to determine content of DNA, collagen, elastin and sulfated GAGs. $\mathrm{n} \geq 3$ for all measures, with standard errors indicated.

${ }^{*} \mathrm{p}<0.01$ compared to native and - indicates not measured. Relative values for native and CHAPS decellularized lung were presented in a prior publication [Petersen et al., 2010].

\section{Collagen and Elastin Content}

Collagen is the key ECM component responsible for the UTS of the lung. As shown in table 1, collagen content in decellularized scaffolds that were prepared using CHAPS decellularization is similar to that of native lung. In contrast, lungs decellularized using SDS detergent, which is anionic and is known to denature proteins more potently than CHAPS [Bodnar et al., 1986; Gilbert et al., 2006], exhibited significantly decreased collagen as compared to native lung tissues, with $787 \pm 102 \mu \mathrm{g}$ collagen per lung, versus $3,480 \pm 103 \mu \mathrm{g}$ for native and 4,220 \pm $480 \mu \mathrm{g}$ for decellularized lung (data for native and CHAPS-treated lung were presented as relative values in Petersen et al. [2010]). This loss of collagen with SDSbased decellularization correlated with decreased UTS, which was not observed in the setting of CHAPS-based decellularization and retained collagen (table 1) [Petersen et al., 2010]. In contrast to collagen, elastin content was somewhat diminished in CHAPS-decellularized scaffolds, as demonstrated by both quantitative assay and histological staining (table 1, fig. 3). In SDS-decellularized tissues, elastin content was also decreased compared to native, with only $1,335 \pm 29.4 \mu \mathrm{g}$ of elastin remaining, compared to $9,980 \pm 826 \mu \mathrm{g}$ for native lung $(\mathrm{p}<0.01)$. Elastin content of SDS-decellularized tissues was not significantly different from CHAPS-treated lungs (table 1). Elastin fibers are critical for the natural recoil of lung tissue, allowing passive exhalation of lung after inspiration with air. The retention of at least some elastin fibers after the decellularization process is therefore important for retention of physiological compliance.

\section{Proteoglycan Content}

Proteoglycans consist of a core protein linked to one or more GAG chains. Most GAGs are sulfated, enabling their detection via quantitative assay. The GAG content of the decellularized scaffolds is significantly lower than that of native lung (about $20 \%$ of native lung levels, table 1). Proteoglycans are found either on the cell surface or within the ECM [Ferdous and Grande-Allen, 2007]. Hence, depletion of GAGs is due in part to the removal of cell-bound GAGs. However, the GAGs found within the ECM can also be solubilized during decellularization, due to the alkalinity of the solution and the presence of detergent [Gilbert et al., 2006]. Assays showing GAG depletion are confirmed by Alcian blue histologic staining, showing that the amount of GAGs remaining in the decellularized lung scaffolds is noticeably diminished compared to native lung in both CHAPS- and SDS-treated tissues (table 1, fig. 3) (relative values for native and CHAPS-treated lung were presented in Petersen et al. [2010]).

\section{Mechanical Characterization}

Mechanical testing of lung tissue strips was used to evaluate the mechanics of both native and decellularized lung samples. The elastic regions of the stress-strain curves indicate that both native and decellularized samples exhibit hysteresis that is similar between the two sample types (fig. 4a). Hysteresis demonstrates that lung is a viscoelastic material, and the difference between the expanding and relaxing curves represents energy that is not recovered during relaxation. Decellularized lung samples also do not exhibit 'creep'. If lung tissue were to creep, it would not deflate to its original position after inflation; thus, the lung would not fully deflate with each inhalation and the lung would progressively expand. This preservation of appropriate elastic lung behavior is important for a lung scaffold; the loss of pulmonary elasticity is seen in several disease states, notably emphysema and pulmonary fibrosis [Gelb et al., 2002]. Although the 

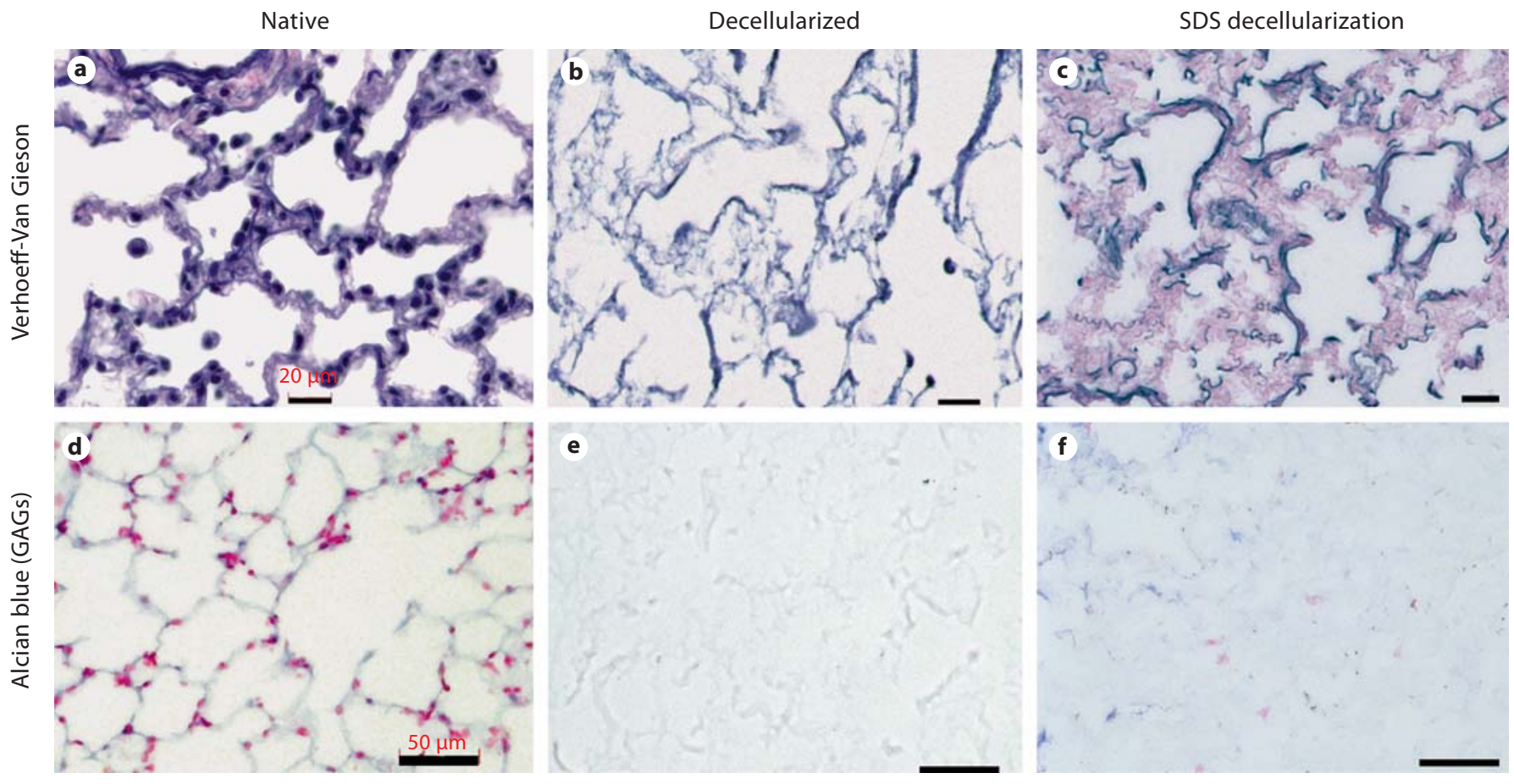

Fig. 3. Histochemical staining of native and decellularized lung. a-c Verhoeff-van Gieson staining for elastin in native lung (a), CHAPS-decellularized lung (b), and SDS-decellularized lung (c) shows retained but diminished elastin fibers (black). d-f Alcian blue staining for GAGs (blue) in native lung (d), CHAPS-decellularized lung (e) and SDS-decellularized lung (f) shows loss of GAGs in both CHAPS- and SDS-decellularized lung (blue). Scale bars in a-c are $20 \mu \mathrm{m}$ and in $\mathbf{d - f}$ are $50 \mu \mathrm{m}$.
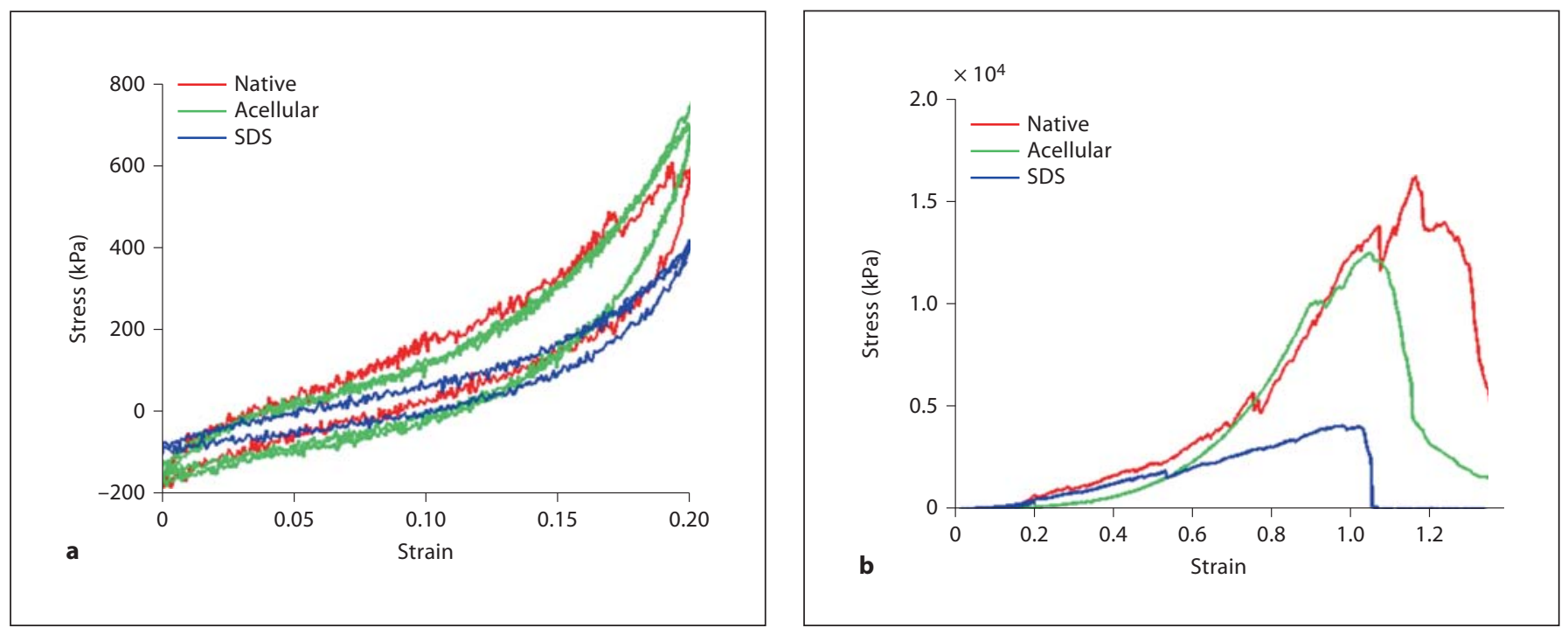

Fig. 4. Stress-strain curves. a Elastic cycling of tissue strips demonstrates hysteresis in native (red), CHAPStreated decellularized (green) and SDS-treated decellularized lung (blue). b Tensile strength testing indicates similar curve profiles for native and CHAPS-treated decellularized lung, but weaker tissue with lower ultimate tensile stress after SDS treatment. 
lungs that were decellularized with CHAPS do lose $60 \%$ of native elastin content, the remaining elastin is apparently sufficient to allow elastic recoil [Petersen et al., 2010]. Tissues that were decellularized using SDS demonstrate similar behavior, although SDS-treated tissues appear to demonstrate less hysteresis (fig. 4a). This correlates with decreased elastin in SDS-treated lungs compared to CHAPS treatment (table 1).

UTS is the stress on a sample at failure, and is a measure of the strength of the material. The UTS of samples decellularized using CHAPS is similar to that of native samples $(19.8 \pm 2.4 \mathrm{kPa}$ for native, $22.3 \pm 1.8 \mathrm{kPa}$ for acellular; table 1) [Petersen et al., 2010]. If samples are decellularized in buffers containing SDS, however, mechanical integrity is compromised, with UTS falling to $7.48 \pm 1.5 \mathrm{kPa}$ (fig. $4 \mathrm{~b}$, table 1). Indeed, the loss of collagen on quantitative assay correlates with loss of UTS. This is not surprising since it is known that SDS can degrade collagen, causing fragmentation and swelling of tissues [Bodnar et al., 1986; Gilbert et al., 2006]. SDS has also been shown to increase tissue extensibility [Mirsadraee et al., 2006].

\section{Model of Observed Mechanical Behavior}

In order to understand the behavior of the decellularized matrices, data were fit to a previously developed theoretical model of the lung parenchyma [Maksym and Bates, 1997]. The model is briefly summarized here, but the reader is referred to Maksym and Bates [1997] for further details.

Measured stress-strain data for lung tissues were fit to the following exponential relationship:

$$
\sigma=G\left(e^{\varepsilon / H}-1\right)
$$

where $\sigma$ is stress, $\varepsilon$ is strain, and $G$ and $H$ are empirically fit parameters.

The lung tissue is modeled as a linear series of elements, each of which is a spring in parallel with a string (fig. 1). The string, which models collagen fibers, exerts no opposing force except when stretched to its stop length $l_{i}$, after which it cannot extend further. The spring models elastic fibers and behaves like a Hookean spring with constant $k_{i}$. We first analyzed the collagen fiber network of the tissue sample, by assuming that each elastin fiber has the same spring constant, $k$. Then, the distribution of stop lengths is represented by $N(l)$, and so the total length of the tissue is

$$
L(l)=L_{r}+\int_{0}^{\infty} l N(l) d l
$$

where $L_{r}$ is the tissue length at rest.
Following a brief derivation, explained in detail in Maksym and Bates [1997], we obtain the following relation for $N(l)$ :

$$
N(l)=\frac{L_{r}}{\left(l+\frac{G A_{r}}{k}\right)^{2}}
$$

where

$$
k=\frac{A_{r} G X}{L_{r} H}
$$

and where $A_{r}$ is the cross-sectional area of the tissue sample, $X$ is the total area under the stop length density distribution $N(l)$, which is taken as 10,000 in Maksym and Bates [1997] and is also used here. Thus, we have a representation for $N(l)$ in terms of measurable parameters, where $A_{r}$ and $L_{r}$ are the measured sample dimensions, and $G$ and $H$ are the fit parameters obtained from the stress-strain curve.

To analyze elastin behavior, we go back to the model and make the simplifying assumption that the collagen fibers all have identical stop lengths $l$. We then obtain a relation for the distribution of spring constants $\mathrm{k}_{\mathrm{i}}, M(k)$. We follow a similar derivation to obtain

$$
M(k)=\frac{k H L_{r} / l}{\left(k+G A_{r} / l\right)^{2}}
$$

where $l=\varepsilon_{f} L_{r} / Y$, where $\varepsilon_{f}=2$ is the maximum strain of the model and $Y$ is the area under the curve of the elastic spring constant distribution. As above and in accordance with Maksym and Bates [1997], we take $Y$ as 10,000. This therefore provides an expression for $M(k)$ in terms of measured parameters and the fit parameters $G$ and $H$. Thus, for a given strip of lung tissue, knowing the stressstrain curve and the tissue dimensions, we can obtain distributions of the stop lengths for the collagen fibers and the spring constants of the elastin fibers, within the framework of the model utilized.

We computed the theoretical distribution of collagen fiber stop lengths, $N(l)$, for native and decellularized lung tissue, based on stress-strain data obtained for both tissues (fig. 5). Each curve is generated from a separate sample of native, CHAPS-decellularized, or SDS-treated lung. Both the native and the CHAPS-treated decellularized lungs show similar stop length distributions, consistent with the similar stress-strain data. However, in analyzing the SDS-treated lungs, there was a trend towards shorter stop lengths, although this difference was not significant ( $p=0.15$ using a Student's t test on the asymptotes). We hypothesize this decrease in stop lengths is due 

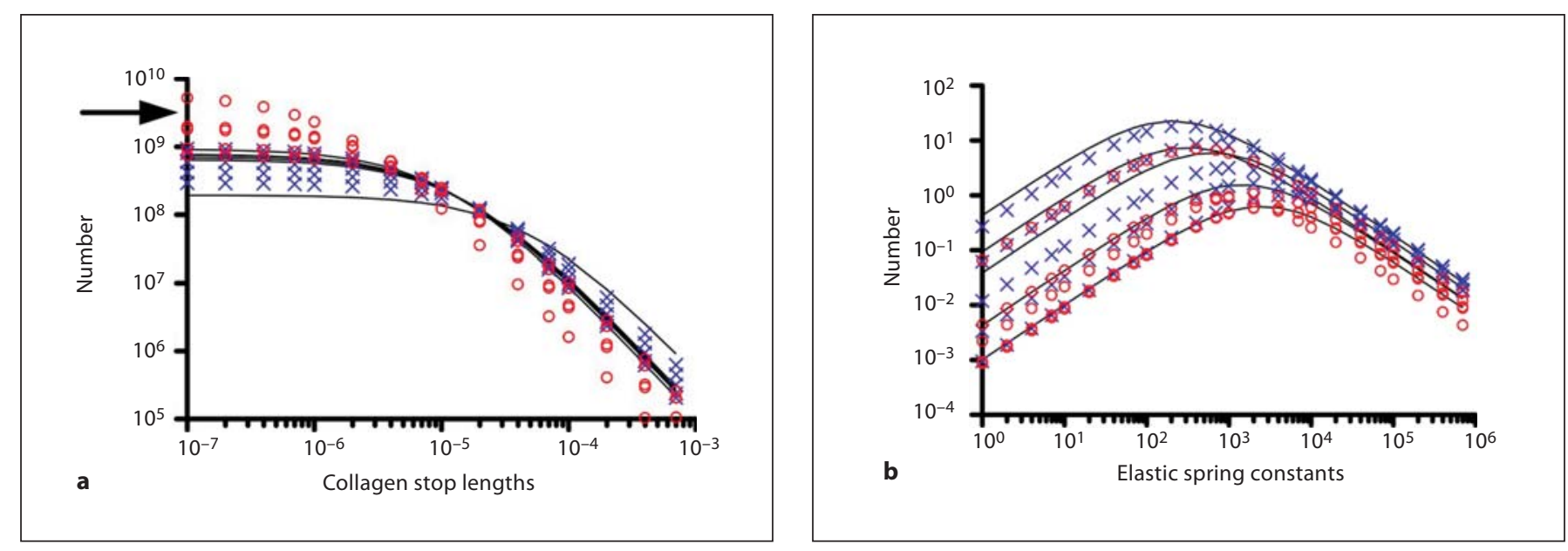

Fig. 5. Modeling studies of lung tissue strips. Solid lines are native lung samples, blue $\times$ marks are CHAPStreated decellularized lung samples, and red circles are SDS-treated decellularized lung samples ( $\mathrm{n}=5 \mathrm{for}$ each). a Distribution of collagen fiber stop lengths showing similar distribution of stop lengths for native and acellular lung, with a nonsignificant trend toward shorter stop lengths in SDS-treated lung (arrow). b Distribution of elastic spring constants are similar in all tissues, though tend to lower numbers overall for SDS-treated lung.

to partial collagen degradation and removal caused by SDS treatment, thus effectively shortening the remaining collagen fibers. Collagen degradation and removal would be facilitated by the SDS-induced collagen fiber denaturation, and is consistent with the decreased overall collagen content of the SDS-treated tissues.

The distribution of elastic spring constants for native and decellularized lung samples was also computed (fig. 5b). No significant differences were detected for any samples, including those decellularized with SDS, though there appeared to be a trend toward fewer elastin fibers across all spring constants for SDS-treated lungs. The behavior of native lung samples is consistent with prior analysis by Maksym et al. [2007]. The findings with the model are therefore consistent with the mechanical testing results. Though a quantitative assay revealed that only $40 \%$ of elastin was retained in CHAPS-treated lungs, only $15 \%$ was retained in SDS-treated lungs, which was likely reflected in the modeling results for elastin fiber numbers (fig. 5b). However, even though substantial elastin is lost by both methods, some evidence of hysteresis remains on mechanical testing (fig. 4a). More detailed modeling studies, together with more advanced mechanical testing, may be needed to identify functional differences between the elasticity of native and decelluarized tissues.

In summary, lungs that are decellularized with CHAPS-based solutions retained collagen matrix by bio- chemical assay, and retained the ultimate mechanical characteristics of native lung. However, SDS-treated lungs retained only about $20 \%$ of native collagen levels and about $15 \%$ of native elastin levels, and demonstrated reduced mechanical strength. In addition, we analyzed the lengths at which each collagen fiber stops extension, and found that this distribution of collagen fiber stop lengths in the CHAPS-decellularized lung resembles that of native lung. In contrast, elastin is depleted by lung matrix decellularization in our hands. However, we analyzed the spring constants for the elastic fibers and found the spring constant distribution does not differ for native and CHAPS-decellularized lung. When taken together, these data demonstrate that the mechanical properties of CHAPS-decellularized lung are comparable to those of native lung.

\section{Discussion}

The collagens are the most important structural component of the lung. Collagen is responsible for the lung's overall mechanical strength, which is derived from three polypeptide $\alpha$-chains that coil around each other to form a right-handed superhelix [Dunsmore and Rannels, 1996]. Elastin is an important ECM component in tissues that require reversible distension, and elastin allows for the intrinsic recoil property of lung tissue. Elastin is rich 
in hydrophobic amino acids and scarce in acidic residues, with the hydrophobic regions believed to be important in conferring its elastic properties [Dunsmore and Rannels, 1996]. Elastin fibers combine with microfibrils to make up the functional elastic fibers of the lung, which are very extensible and maintain a linear stress-strain relationship out to about $200 \%$ strain [Kreis and Vale, 1999]. Proteoglycans are proteins that are posttranslationally modified with GAGs. They are found on cell surfaces, within intracellular vesicles, and incorporated into the ECM [Kreis and Vale, 1999]. GAGs help control macromolecular and cellular movement across the basal lamina [Kreis and Vale, 1999], and may play a role in the mechanical integrity of the lung [Cavalcante et al., 2005].

The development of a decellularized lung matrix is important as a scaffold for tissue-engineering applications. By removing cellular material from the lung, we are able to probe the contributions of the various ECM components to lung mechanics in the absence of cellular influence. We observed the removal of virtually all DNA from decellularized lung matrices. The complete removal of DNA is very difficult due to the high negative charge of the molecule and its inherent 'stickiness' to protein matrices. The removal of cellular material is also evident based on histological staining and electron microscopy.

We have previously demonstrated that collagen is retained in CHAPS-decellularized lung matrices via a quantitative assay and mechanical testing, as well as histochemical and immunofluorescent staining [Petersen et al., 2010]. Here, we provide further data to support the retention of collagen in CHAPS-treated lungs and contrast this with loss of collagen in SDS-decellularized tissues. Mechanical testing indicates that the CHAPSdecellularized scaffolds retain hysteresis, or path dependence, during cyclic stress, although the degree of hysteresis is reduced in SDS-treated lungs as compared to native or CHAPS-treated lungs (fig. 4a). Hysteresis behavior is analogous to (but quite distinct from) pressurevolume curves of whole lung, where hysteresis is also important to the macroscopic function of the lung, although in this case it is largely due to the effects of alveolar recruitment [Escolar and Escolar, 2004]. The decellularized scaffolds also do not demonstrate creep upon elastic testing, and relax to the same stress after application of strain. If the scaffolds exhibited creep, then with every expansion (strain application) the tissue would not relax fully but would return to a slightly stretched state. Clearly, for tissue-engineering purposes, ensuring that lung scaffolds do not creep with successive inflations will be critical for any in vivo implantation.
Cyclic mechanical distension, without creep, is important in aiding the differentiation of type I pneumocytes [Gutierrez et al., 1998; Dobbs and Gutierrez, 2001] and the final differentiation of type II pneumocytes [Inanlou and Kablar, 2005b]. During fetal development, fetal breathing movements are important for the advanced development of pulmonary tissue [Inanlou and Kablar, 2005a]. In the absence of fetal breathing movements, type I pneumocytes cannot undergo late differentiation and thus cannot flatten along the alveolar surface [Inanlou and Kablar, 2005a]. The clinical result of a lack of fetal breathing movements is pulmonary hypoplasia, a common neonatal disorder that has been found in $15 \%$ of neonatal autopsies [Wigglesworth and Desai, 1982]. In order to successfully develop engineered lung tissues, the lung scaffold must be able to withstand such physiological movements and pressures, further emphasizing the importance of the mechanical integrity of the lung scaffolds. The data presented herein suggest that decellularized scaffolds will be able to withstand physiological movements, and that such scaffolds are therefore promising for use in tissue-engineering applications, although further characterization is still needed.

Decellularization using SDS, in our hands, significantly degrades the mechanical strength of the decellularized matrices. This may be related to the amphipathic structure of SDS, allowing it to bind water and increase tissue water content [Bodnar et al., 1986]. Work by Cortiella et al. [2010] on lung decellularization using SDScontaining solutions demonstrated a loss of collagen type IV, the principal basement membrane collagen in the lung periphery, although collagen type I was preserved. Other studies have not always demonstrated a decrease in UTS with SDS treatment [Mirsadraee et al., 2006], although this may be due to tissue and methodological differences. Mirsadraee et al.[2006] found collagen retention in SDS-treated pericardial tissue, which contains much more densely packed collagen fibers than does the lung.

We have used a simple model of peripheral lung matrix to further investigate our findings. We modeled the collagen and elastin components of the lung matrix, and found our modeling results to be comparable with our mechanical testing and quantitative assay results for native and decellularized lung. However, a direct correlation between the organization of collagen and elastin fibers and overall lung tissue characteristics, such as pressure-volume relationships or tensile strength, is not possible [Suki et al., 2005]. When collagen is degraded and removed via decellularization with SDS, the effects 
of this are apparent in the model, in which both the number and length of collagen fibers are diminished.

Collagen and elastin are generally accepted to be the principal determinants of the mechanical properties of a lung matrix. This is supported by findings in this study, whereby the mechanics of native and decellularized lung are similar when collagen and some elastin are preserved while proteoglycans and cells are removed. However, proteoglycans can contribute to the mechanics of lung tissue in smaller ways, as these hydrophilic molecules can resist compression and shear [Buschmann and Grodzinsky,
1995]. Cavalcante et al. [2005] noted small changes in the stress-strain curves of lung tissue with changes in the tonicity of the surrounding liquid, and furthermore noted that these changes were reduced after proteoglycan digestion. Therefore, proteoglycans play at least some role in the mechanics of the lung matrix. In this study, where proteoglycans are largely removed from the decellularized matrices, no significant effect of their removal was noted. Future studies are needed to explore this area in greater depth to investigate proteoglycan contributions to lung matrix mechanics.

\section{References}

Bader, A., T. Schilling, O.E. Teebken, G. Brandes, T. Herden, G. Steinhoff, A. Haverich (1998) Tissue engineering of heart valves - human endothelial cell seeding of detergent acellularized porcine valves. Eur J Cardiothorac Surg 14: 279-284.

Badylak, S.F., G.C. Lantz, A. Coffey, L.A. Geddes (1989) Small intestinal submucosa as a large diameter vascular graft in the dog. J Surg Res 47: 74-80.

Bates, J.H., G.S. Davis, A. Majumdar, K.J. Butnor, B. Suki (2007) Linking parenchymal disease progression to changes in lung mechanical function by percolation. Am J Resp Crit Care Med 176: 617-623.

Bodnar, E., E.G. Olsen, R. Florio, J. Dobrin (1986) Damage of porcine aortic valve tissue caused by the surfactant sodiumdodecylsulphate. Thorac Cardiovasc Surg 34: 82-85.

Buschmann, M.D., A.J. Grodzinsky (1995) A molecular model of proteoglycan-associated electrostatic forces in cartilage mechanics. J Biomech Eng 117: 179-192.

Cavalcante, F.S., S. Ito, K.K. Brewer, H. Sakai, A.M. Alencar, M.P. Almeida, J.S. Andrade, A. Majumdar, E.P. Ingenito, B. Suki (2005) Mechanical interactions between collagen and proteoglycans: implications for the stability of lung tissue. J Appl Physiol 98: 672679.

Cortiella, J., J. Niles, A. Cantu, A. Brettler, A. Pham, G. Vargas, S. Winston, J. Wang, S. Walls, J. Nichols (2010) Influence of acellular natural lung matrix on murine embryonic stem cell differentiation and tissue formation. Tissue Eng 16: 2565-2580.

Dahl, S.L.M., J. Koh, V. Prabhakar, L.E. Niklason (2003) Decellularized native and engineered arterial scaffolds for transplantation. Cell Transplant 12: 659-666.

Dobbs, L.G., J.A. Gutierrez (2001) Mechanical forces modulate alveolar epithelial phenotypic expression. Comp Biochem Physiol A 129: 261-266.
Dunsmore, S.E., D.E. Rannels (1996) Extracellular matrix biology in the lung. Am J Physiol 270: L3-L27.

-Escolar, J.D., A. Escolar (2004) Lung hysteresis: a morphological view. Histol Histopathol 19: 159-166.

Ferdous, Z., K.J. Grande-Allen (2007) Utility and control of proteoglycans in tissue engineering. Tissue Eng 13: 1893-1904.

Foronjy, R., T. Nkyimbeng, A. Wallace, J. Thankachen, Y. Okada, V. Lemaitre, J. D'Armiento (2008) Transgenic expression of matrix metalloproteinase- 9 causes adult-onset emphysema in mice associated with the loss of alveolar elastin. Am J Physiol Lung Cell Mol Physiol 294: L1149-L1157.

Gelb, A.F., J. Licuanan, C.M. Shinar, N. Zamel (2002) Unsuspected loss of lung elastic recoil in chronic persistent asthma. Chest 121: 715721.

Gilbert, T.W, T.L. Sellaro, S.F. Badylak (2006) Decellularization of tissues and organs. Biomaterials 27: 3675-3683.

Grant, R.A. (1964). Estimation of hydroxyproline by the autoanalyser. J Clin Pathol 17: 685-686.

Gutierrez, J.A, R.F. Gonzalez, L.G. Dobbs (1998) Mechanical distension modulates pulmonary alveolar epithelial phenotypic expression in vitro. Am J Physiol 274: L196-L202.

Inanlou, M.R., B. Kablar (2005a) Abnormal development of the intercostal muscles and the rib cage in Myf5-/- embryos leads to pulmonary hypoplasia. Dev Dyn 232: 43-54.

Inanlou, M.R., B. Kablar (2005b) Contractile activity of skeletal musculature involved in breathing is essential for normal lung cell differentiation, as revealed in Myf5-/-: MyoD-/- embryos. Dev Dyn 233: 772-782.

Kreis, T., R. Vale (1999) Guidebook to the Extracellular Matrix, Anchor, and Adhesion Proteins. Oxford, Oxford University Press.
Maksym, G.N., J.H.T. Bates (1997) A distributed nonlinear model of lung tissue elasticity. J Appl Physiol 82: 32-41.

McFetridge, P.S., J.W. Daniel, T. Bodamyali, M. Horrocks, J.B. Chaudhuri (2004) Preparation of porcine carotid arteries for vascular tissue engineering applications. J Biomed Mat Res A 70: 224-234.

Mirsadraee, S., H.E. Wilcox, S.A. Korossis, J.N. Kearney, K.G. Watterson, J. Fisher, E. Ingham (2006) Development and characterization of an acellular human pericardial matrix for tissue engineering. Tissue Eng Part A 12: 763-773.

Ott, H.A., B. Clippinger, C. Conrad, C. Schuetz, I. Pomerantseva, I. Ikonomou, D. Kotton, J.P. Vacanti (2010) Regeneration and orthotopic transplantation of a bioartificial lung. Nat Med 16: 927-933.

Petersen, T.H., E.A. Calle, L. Zhao, E.J. Lee, L. Gui, M.B. Raredon, K. Gavrilov, T. Yi, Z.W. Zhuang, C. Breuer, E. Herzog, L.E. Niklason (2010) Tissue-engineered lungs for in-vivo implantation. Science 329: 538-541.

- Price, A., K. England, A. Matson, B. Blazar, A. Panoskaltsis-Mortari (2010) Development of a decellularized lung bioreactor system for bioengineering the lung: the matrix reloaded. Tissue Eng 16: 2581-2591.

- Sasaki, N, S. Odajima (1996) Stress-strain curve and Young's modulus of a collagen molecule as determined by the $\mathrm{X}$-ray diffraction technique. J Biomech 29: 655-658. (http://linkinghub.elsevier.com/retrieve/pii/ 0021929095001107).

- Suki, B., S. Ito, D. Stamenovic, K.R. Lutchen, E.P. Ingenito (2005) Biomechanics of the lung parenchymal: critical roles of collagen and mechanical forces. J Appl Physiol 98: 18921899.

Wigglesworth, J.S., R. Desai (1982) Is fetal respiratory function a major determinant of perinatal survival? Lancet $i$ : 264-267. 